\title{
Low-Power Temperature Sensor with Complementary-Metal-Oxide-Semiconductor Circuits
}

\author{
Shu-Chung $\mathrm{Yi}^{*}$ \\ Department of Computer Science and Information Engineering, National Changhua University of \\ Education, Changhua City, 500 Taiwan, R.O.C.
}

(Received November 5, 2012; accepted February 4, 2013)

Key words: CMOS sensor, PTAT, subthreshold region, temperature sensor

A low-power smart temperature sensor is proposed in this paper. The sensor was composed of a proportional-to-absolute-temperature (PTAT) circuit and a ring oscillator. The PTAT circuit was used to generate a temperature-related current. The current was used to drive the ring oscillator, which may generate a temperature-related oscillated signal. The sensor was implemented by the $0.35 \mu \mathrm{m} 2 \mathrm{P} 4 \mathrm{M}$ TSMC complementarymetal-oxide-semiconductor (CMOS) process technology. The power consumption is about $195.15 \mathrm{nW}$. The linearity of the output frequency versus temperature is marked by the $R$-square rule. The value of the linearity is 0.98 in the entire temperature range. The proposed sensor requires only one supply voltage of $3.3 \mathrm{~V}$. The core area of the sensor is small enough for combining itself with other circuits that require temperature monitoring.

\section{Introduction}

In the near future, information network systems with diverse smart sensors will be developed and distributed all over the world to make infrastructures for the information age. Such network systems require a great number of sensors that measure several physical parameters in the environment. ${ }^{(1-3)}$

These sensors must operate with ultralow power because they will likely be arranged under conditions where they will have to obtain essential energy from poor energy sources, such as microbatteries and solar cells. ${ }^{(4)}$

Traditional sensors have the ability to sense environment parameters, but they do not have signal processing and control functions. The instrument industry did not propose the concept of a smart sensor until the seventies and it devised a way to put the process and control of the sense signal into a single sensor element.

The sensor was called smart sensor in America and intelligent sensor in Europe. The smart sensor is constructed with the logic function, control function, and perceive

*Corresponding author: e-mail: scyi@cc.ncue.edu.tw 
element in the same chip using integration technology, so the smart sensor is better than the conventional sensor, whether in the measurement or diverse function of the smart sensor. ${ }^{(5)}$

Temperature sensors have become common elements in a broad range of recent integrated circuits. They are used for measurements of temperature and indirectly for power management of physical, biomedical, and industrial applications.

In the smart temperature sensor, the voltage or current source was applied into the analog-to-digital converter (ADC). The output of the temperature sensor generated a digital output, such as an oscillated square wave. Thus, the conventional smart temperature sensor can be implemented by using the temperature sensor and ADC. The block diagram of the conventional smart temperature sensor ${ }^{(6)}$ is shown in Fig. 1.

The proposed smart temperature sensor is composed of only one voltage source. The sensor requires 19 MOSFETs. ${ }^{(7)}$ The power consumption is $195.15 \mathrm{nW}$. The conventional smart temperature sensor is composed of at least two power sources. The advantages of low power and small size make the sensor easily combine with other circuits that require temperature information.

\section{Temperature Sensor}

Figure 2 depicts the schematic of the temperature sensor. The sensor is based on a nine-transistor temperature-dependent current source. The output voltage of the threshold reference $\left(\mathrm{M}_{1}-\mathrm{M}_{7}\right)$ appears on nodes $\mathrm{C}$ and $\mathrm{D}$ and their values are described as follows:

$$
\begin{aligned}
& V_{\mathrm{C}}=V_{\mathrm{T}}\left\{1+\frac{\sqrt{\frac{W_{1} / L_{1}}{W_{2} / L_{2}}}}{\left.\sqrt{\frac{W_{1} / L_{1}}{W_{2} / L_{2}}}-\sqrt{\frac{W_{5} / L_{5}}{W_{7} / L_{7}}}-\sqrt{\frac{W_{5} / L_{5}}{W_{6} / L_{6}}}\right\},}\right. \\
& V_{\mathrm{D}}=V_{\mathrm{T}}\left\{1+\frac{\sqrt{\frac{W_{5} / L_{5}}{W_{7} / L_{7}}}}{\sqrt{\frac{W_{1} / L_{1}}{W_{2} / L_{2}}}-\sqrt{\frac{W_{5} / L_{5}}{W_{7} / L_{7}}}-\sqrt{\frac{W_{5} / L_{5}}{W_{6} / L_{6}}}}\right\},
\end{aligned}
$$

Smart Temperature Sensor

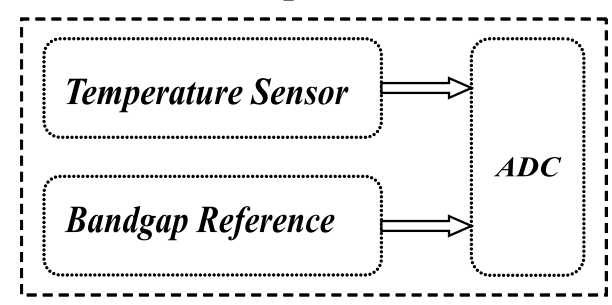

Fig. 1. Smart temperature sensor. 


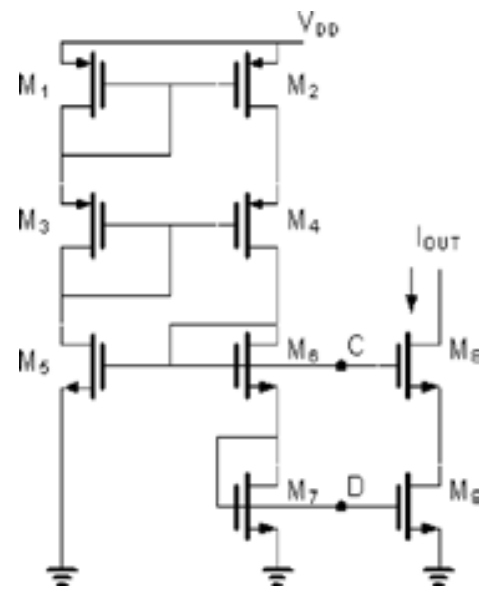

Fig. 2. Detailed circuit of the temperature sensor.

where $V_{\mathrm{T}}$ is the threshold voltage of MOSFET. $W_{i}$ and $L_{i}$ are the channel width and channel length of MOSFET $\mathrm{M}_{i}$, as shown in Fig. 2, respectively. From eqs. (1) and (2), it is obvious that $V_{\mathrm{C}}$ and $V_{\mathrm{D}}$, as shown in Fig. 2, depend only on the threshold voltage $V_{\mathrm{T}}$ and the geometric ratios of the transistor channel sizes. The voltage $V_{\mathrm{D}}$ controls the gate of the transistor $\mathrm{M}_{9}$, yielding the drain current $I_{\mathrm{OUT}}$ described as

$$
I_{\mathrm{OUT}}=\beta_{9}\left(V_{\mathrm{T}}\right)^{2}\left\{1+\frac{\sqrt{\frac{W_{1} / L_{1}}{W_{2} / L_{2}}}}{\sqrt{\frac{W_{1} / L_{1}}{W_{2} / L_{2}}}-\sqrt{\frac{W_{5} / L_{5}}{W_{7} / L_{7}}}-\sqrt{\frac{W_{5} / L_{5}}{W_{6} / L_{6}}}}\right\}^{2}=(\text { const. }) \beta_{9}\left(V_{\mathrm{T}}\right)^{2},
$$

where $\beta_{9}$ is the gain factor of MOSFET $\mathrm{M}_{9}$. The temperature dependence of the output current can be expressed as

$$
\frac{\Delta I_{\text {OUT }}}{I_{\text {OUT }}}=\left\{\frac{1}{\beta} \frac{\mathrm{d} \beta}{\mathrm{d} T}+\frac{2}{V_{\mathrm{T}}} \frac{\mathrm{d} V_{\mathrm{T}}}{\mathrm{d} T}\right\} \Delta T
$$

The dependences of threshold voltage $V_{\mathrm{T}}$ and gain factor $\beta$ are on the order of

$$
\begin{aligned}
& \frac{\Delta I_{\mathrm{OUT}}}{\Delta T} \approx-1.8 \mathrm{mV} / \mathrm{K}, \\
& \frac{1}{\beta} \frac{\Delta \beta}{\Delta T} \approx-0.5 \% / \mathrm{K} .
\end{aligned}
$$


Therefore, the output current shows a temperature dependence of about 0.9 to $1 \%$ and can be regarded as a temperature signal. A current-to-frequency converter ${ }^{(8)}$ also converts the temperature signal to a digital signal. The temperature-dependent current $I_{\text {OUT }}$ provides the current supply of a ring oscillator to generate a temperature-related square wave.

\section{Ring Oscillator}

In a simple ring oscillator, the total number of inverters must be odd, as shown in Fig. 3. For example, when the ring oscillator is implemented by a five-stage inverter chain, the ring oscillator can provide a frequency of $\left(1 / 10 t_{\mathrm{D}}\right) \cdot{ }^{(9)}$

An oscillator is used as an element to convert signals from analog to digital. The relationship between output frequency and temperature is shown as follows: ${ }^{(10)}$

$$
\begin{gathered}
f_{\text {out }}=\frac{1}{2 N t_{\mathrm{D}}}=\frac{\mu_{n} C_{\mathrm{OX}}\left(V_{\mathrm{DD}}-V_{\mathrm{TN}}\right)}{2 N\left(\frac{L}{W}\right)_{n} C_{\mathrm{L}}} \frac{1}{\ln \left(\frac{1.5 V_{\mathrm{DD}}-2 V_{\mathrm{TN}}}{0.5 V_{\mathrm{DD}}}\right)}, \\
=\frac{\mu_{0}\left(\frac{T}{T_{0}}\right)^{k m}\left[V_{\mathrm{DD}}-V_{\mathrm{TP}}+|\alpha|\left(T-T_{0}\right)\right]}{2 N\left(\frac{L}{W}\right)_{n} C_{\mathrm{L}}} \frac{1}{\ln \left\{3-\frac{\left[V_{\mathrm{TP}}-|\alpha|\left(T-T_{0}\right)\right]}{0.5 V_{\mathrm{DD}}}\right\}},
\end{gathered}
$$

where $V_{\mathrm{TN}}=V_{\mathrm{TP}}=V_{\mathrm{T}}$, i.e., the rising time $\left(t_{\mathrm{PLH}}\right)$ is assumed to be the same as the falling time $\left(t_{\mathrm{PHL}}\right)$.

\section{Construction of Proposed Proportional-to-Absolute Temperature (PTAT)}

The main requirements for the circuit design are low cost and low power consumption. As the intelligent network system becomes readily accessible, there is an increasing opportunity for incorporating the sensor chip into the system-on-chips.

The sensor chip has even been applied in the radio frequency identification (RFID). It is therefore important to consider the surface area and power consumption of a sensor chip. The circuits can be divided into two parts: a PTAT current-generating circuit and a current-driving ring oscillator. Figure 4 depicts the construction of the smart sensor. The PTAT subcircuits provide a supply current to drive the rear ring oscillator. The current generated by PTAT is microampere and is proportional to temperature. By doing so, the oscillator will generate an output wave that is absolutely temperature-related.

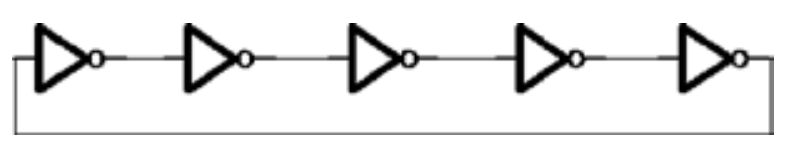

Fig. 3. Five-stage ring oscillator. 
Smart temperature sensor

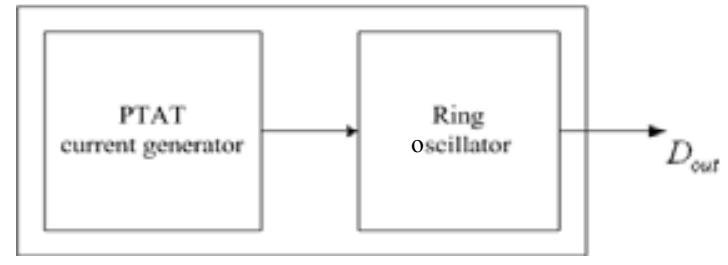

Fig. 4. Block diagram of the proposed temperature sensor.

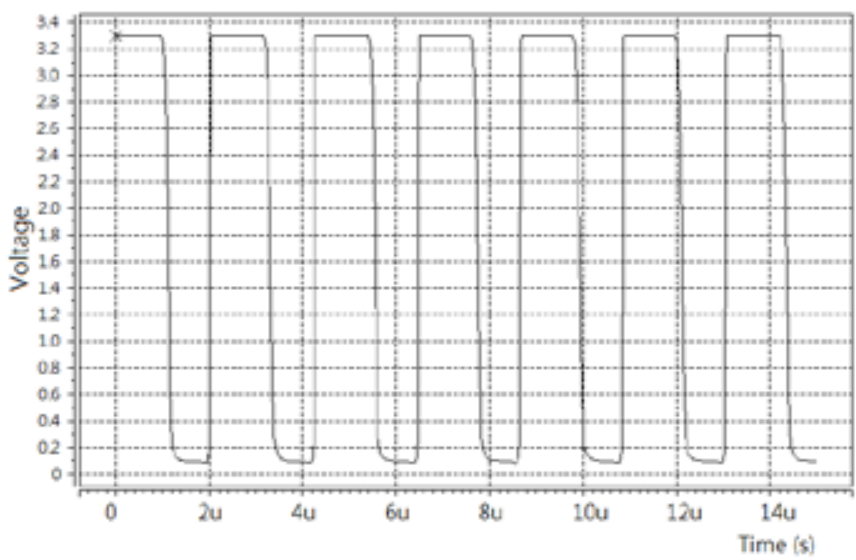

Fig. 5. Output waveform of the ring oscillator.

\section{Simulation Results and Implementations}

Figure 5 shows the output of this proposed circuit when the temperature is $25^{\circ} \mathrm{C}$ in postsimulation. From the waveform results, we can observe that the sensor can still work properly. Figure 6 shows the relationship between the PTAT current and temperature. From Fig. 6, it is obvious that PTAT current has good linearity in the valid temperature range. An $R$-square value of 0.98 has been reached. Therefore, the simulation result can be acceptable.

The layout of the power source is separated into analog and digital regions because of noise reduction. The approach prevents the noise generated by the digital circuit from affecting the analog circuit. In addition, a guard ring is placed on the periphery of the analog circuit for resisting external noises. 


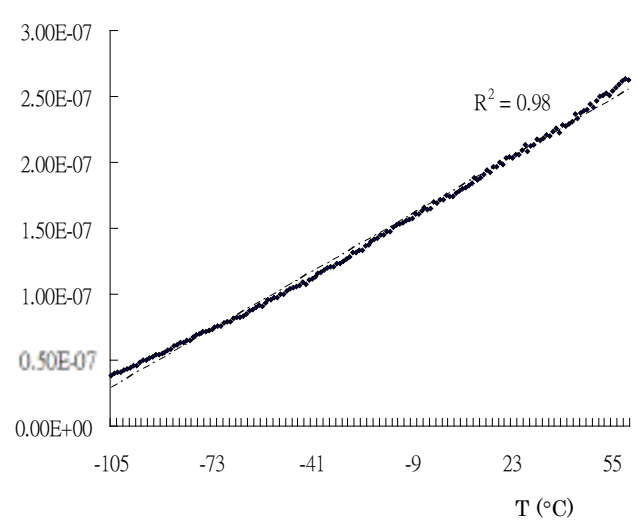

Fig. 6. Linearity of PTAT current vs temperature.

\section{Conclusions}

To reflect the demand of low cost and low power, a smart temperature sensor is successfully developed. The sensor circuit uses the PTAT connected to only one power supply. The ring oscillator generates the oscillated signal proportional to temperature. The approach is simpler than the convention one and the approach can reduce the complexity of this sensor.

The smart temperature sensor was fabricated by the $0.35 \mu \mathrm{m}$ 2P4M TSMC CMOS process technology. The core area is only $1105.59 \mu \mathrm{m}^{2}$. The temperature sensitivity range is about $165{ }^{\circ} \mathrm{C}$ and the power consumption is about $159.15 \mathrm{nW}$. It has an $R$-square value of 0.98 . Therefore, a low-power and low-cost temperature sensor is realized.

\section{References}

1 H. C. Hsiao, H. C. Wen and M. Nakano: IJETI 2 (2012) 66.

2 T. Nenov and S. Ivanov: Sens. Mater. 2 (2007) 95.

3 P. Enquist: Sens. Mater. 6 (2005) 307.

4 K. Ueno, T. Hirose, T. Asai and Y. Amemiya: Int. Symp. ISPACS, ed. Takao Onoye (IEEE, Japan, 2006) 546.

5 OPTOLINK: smart sensor, http://www.pida.org.tw/optolink/optolink_pdf/87051503.pdf (accessed on March 2012).

6 C. C. Chen, A. W. Liu, Y. C. Chang and P. Chen: IEEE Sens. 46 (2005) 849.

7 V. Székely, C. Márta, Z. Kohári and M. Rencz: IEEE Trans. VLSI Syst. 5 (1997) 270.

8 B. Razavi: Design of Analog CMOS Integrated Circuits, ed. Razavi (McGraw-Hill, New York, 2001) Chap. 3.

9 J. H. Chen: Design of Low Voltage and Low Current Micro-Operation Amplifier Using the Bulk-Driven Technique to Operate in Subthreshold Region, ed. Cheng-Fang Tai (National United University, Taiwan, 2006) Chap. 4.

10 P. H. Huang, H. Lin and Y. T. Lin: IEEE TCAS 53 (2006) 882. 